\title{
THE
}

\section{Dynamics of an Integrable Two-sublattice Spin Model with Long- range Interaction}

Jian-Min Liu

University of Rhode Island

Gerhard Müller

University of Rhode Island, gmuller@uri.edu

Follow this and additional works at: https://digitalcommons.uri.edu/phys_facpubs

Terms of Use

All rights reserved under copyright.

\section{Citation/Publisher Attribution}

Liu, J. M., \& Muller, G. (1991). Dynamics of an integrable two-sublattice spin model with long-range interaction. Physical Review B, 44(21), 12020-12022. doi: 10.1103/PhysRevB.44.12020

Available at: http://dx.doi.org/10.1103/PhysRevB.44.12020

This Article is brought to you for free and open access by the Physics at DigitalCommons@URI. It has been accepted for inclusion in Physics Faculty Publications by an authorized administrator of DigitalCommons@URI. For more information, please contact digitalcommons-group@uri.edu. 


\title{
Dynamics of an integrable two-sublattice spin model with long-range interaction
}

\author{
Jian-Min Liu and Gerhard Müller \\ Department of Physics, The University of Rhode Island, Kingston, Rhode Island 02881-0817
}

(Received 8 July 1991)

\begin{abstract}
The dynamics of the classical two-sublattice $X Y Z$ model with uniform intersublattice interaction and zero intrasublattice interaction is completely integrable for arbitrary system sizes. This makes the system amenable to an exact analysis of dynamic correlation functions. Here we present some exact results for the case with isotropic interaction ( $X X X$ model). The dynamical properties of the two-sublattice $X Y Z$ model are compared with those of the equivalent-neighbor $X Y Z$ model and categorized into universality classes of dynamical behavior.
\end{abstract}

In studies of dynamic correlation functions the focus is, in general, on their long-time asymptotic behavior or, equivalently, on the singularity structure of the associated spectral densities. It turns out to be equally useful and revealing, albeit for different reasons, to analyze the properties of the same spectral densities at high frequencies, specifically their decay law, expressible as

$$
\Phi(\omega) \sim \exp \left(-\omega^{2 / \lambda}\right)
$$

in terms of a characteristic exponent $\lambda$. That decay law governs the growth rate of the sequence of recurrents which determine the relaxation function (Hilbert transform of the spectral density) in the continued-fraction representation. ${ }^{1}$ The value of $\lambda$ contains valuable information on the underlying dynamical processes taking place in the system. In some sense, this information is complementary to that inferred from the long-time asymptotic behavior.

A detailed study of the dynamics of various quantum and classical spin models has prompted us to adopt the concept of universality class for a categorization of dynamical behavior on the basis of the characteristic exponent $\lambda .{ }^{1}$ For the equivalent-neighbor $X Y Z$ model, ${ }^{2-4}$

$$
H=-\frac{1}{2 \sqrt{N}} \sum_{\substack{i, j=1 \\(i \neq j)}}^{N}\left(J_{x} S_{i}^{x} S_{j}^{x}+J_{y} S_{i}^{y} S_{j}^{y}+J_{z} S_{i}^{z} S_{j}^{z}\right),
$$

we were able to demonstrate four different prototype universality classes: $\lambda=0$ (compact support), $\lambda=1$ (Gaussian decay), $\lambda=2$ (exponential decay), $\lambda=3$ (stretched exponential decay). All four universality classes have been interpreted in terms of basic notions of classical dynamics. ${ }^{1}$

Here we present a different spin model for dynamical analysis in this particular context: the two-sublattice $X Y Z$ model with uniform intersublattice interaction and zero intrasublattice interaction:

$H=-\frac{1}{\sqrt{N}} \sum_{l=1}^{N_{A}} \sum_{l^{\prime}=1}^{N_{B}}\left(J_{x} S_{l}^{x} S_{l^{\prime}}^{x}+J_{y} S_{l}^{y} S_{l^{\prime}}^{y}+J_{z} S_{l}^{z} S_{l^{\prime}}^{z}\right)$,

where $N=N_{A}+N_{B}$ is the total number of spins. The special $1 / \sqrt{N}$ scaling of the exchange constants in the two models (2) and (3) guarantees that the intrinsic dynamics, specified by Hamilton's equation, $d \mathbf{S}_{l} / d t$ $=-\mathbf{S}_{l} \times \partial H / \partial \mathbf{S}_{l}$, stays nontrivial in the limit $N \rightarrow \infty$. Note that in a different scaling regime $(1 / N$ instead of $1 / \sqrt{N}$ ), the two models play an important role as microscopic realizations for mean-field models of an $X Y Z$ ferromagnet and an $X Y Z$ two-sublattice antiferromagnet, respectively.

The equations of motion for individual spins of the two-sublattice $X Y Z$ model (3) read

$$
\begin{aligned}
& \dot{S}_{l}^{\alpha}=J_{\gamma} \sigma_{B}^{\gamma} S_{l}^{\beta}-J_{\beta} \sigma_{B}^{\beta} S_{l}^{\gamma}, \quad l=1,2, \ldots, N_{A}, \\
& \dot{S}_{l^{\prime}}^{\alpha}=J_{\gamma} \sigma_{A}^{\gamma} S_{l^{\prime}}^{\beta}-J_{\beta} \sigma_{A}^{\beta} S_{l}^{\gamma}, \quad l^{\prime}=1,2, \ldots, N_{B},
\end{aligned}
$$

with $\alpha \beta \gamma=$ c.p. $(x y z)$. The collective-spin variables

$$
\sigma_{A}=\frac{1}{\sqrt{N}} \sum_{l=1}^{N_{A}} \mathbf{S}_{l}, \quad \sigma_{B}=\frac{1}{\sqrt{N}} \sum_{l^{\prime}=1}^{N_{B}} \mathbf{S}_{l^{\prime}}
$$

represent the vectors of instantaneous sublattice magnetization fluctuations. Summing Eqs. (4) over all sublattice sites and dividing by $\sqrt{N}$ yields the equations of motion for the two sublattice spins,

$\dot{\sigma}_{A}^{\alpha}=J_{\gamma} \sigma_{B}^{\gamma} \sigma_{A}^{\beta}-J_{\beta} \sigma_{B}^{\beta} \sigma_{A}^{\gamma}, \quad \dot{\sigma}_{B}^{\alpha}=J_{\gamma} \sigma_{A}^{\gamma} \sigma_{B}^{\beta}-J_{\beta} \sigma_{A}^{\beta} \sigma_{B}^{\gamma}$,

with $\alpha \beta \gamma=$ c.p. $(x y z)$. These equations describe the nonlinear rotational dynamics of an effective two-spin model:

$$
\bar{H}=-\sum_{\alpha=x y z} J_{\alpha} \sigma_{A}^{\alpha} \sigma_{B}^{\alpha} \text {. }
$$

The integrability of this two-body problem was first proven in a quite different context, through explicit construction of a second independent integral of the motion ${ }^{5}$

$\bar{I}=-\sum_{\alpha \beta \gamma=\text { c.p. }(x y z)} J_{\alpha} J_{\beta} \sigma_{A}^{\gamma} \sigma_{B}^{\gamma}+\sum_{\alpha=x y z} \frac{1}{2} J_{\alpha}^{2}\left[\left(\sigma_{A}^{\alpha}\right)^{2}+\left(\sigma_{B}^{\alpha}\right)^{2}\right]$.

For given solutions $\sigma_{A}(t)$ and $\sigma_{B}(t)$, Eqs. (4) for individual spins turn into a set of linear and decoupled vector equations with time-dependent coefficients.

A complete set of $N$ independent integrals of the 
motion in involution for the two-sublattice $X Y Z$ model (3) consists of two invariants which govern the time evolution of the two vectors $\sigma_{A}$ and $\sigma_{B}$ and of $N-2=\left(N_{A}-1\right)+\left(N_{B}-1\right)$ invariants which govern the time evolution of the individual spins in arrays $A$ and $B$. The first two invariants are $\bar{H}$ and $\bar{I}$, and the remaining $N-2$ can be selected as follows: ${ }^{6}$

$$
\begin{aligned}
& I_{l}^{A}=\sum_{k<l} \mathbf{S}_{l} \cdot \mathbf{S}_{k}, \quad l=2,3, \ldots, N_{A}, \\
& I_{l^{\prime}}^{B}=\sum_{k^{\prime}<l^{\prime}} \mathbf{S}_{l^{\prime}} \cdot \mathbf{S}_{k^{\prime}}, \quad l^{\prime}=2,3, \ldots, N_{B} .
\end{aligned}
$$

It is interesting to compare these properties of the two-sublattice $X Y Z$ model (3) with those of the equivalent-neighbor $X Y Z$ model (2). The latter is not completely integrable except for $N=2$ or $N=\infty$. Only the fully isotropic case $\left(J_{x}=J_{y}=J_{z}, X X X\right.$ model) is completely integrable for arbitrary $N$. For that case, a set of $N$-independent integrals of the motion can be chosen as follows: ${ }^{6}$

$$
\begin{aligned}
I_{1}=\sum_{k=1}^{N} S_{k}^{z}, \quad I_{l}=\mathbf{S}_{l} \cdot\left(\mathbf{S}_{1}+\mathbf{S}_{2}+\cdots\right. & \left.+\mathbf{S}_{l-1}\right), \\
l & =2,3, \ldots, N .
\end{aligned}
$$

The equations of motion for individual spins of Hamiltonian (2) read

$\dot{S}_{i}^{\alpha}=J_{\gamma} \sigma_{\gamma} S_{i}^{\beta}-J_{\beta} \sigma_{\beta} S_{i}^{\gamma}-\frac{1}{\sqrt{N}}\left(J_{\gamma} S_{i}^{\gamma} S_{i}^{\beta}-J_{\beta} S_{i}^{\beta} S_{i}^{\gamma}\right)$

with $\alpha \beta \gamma=$ c.p. $(x y z)$. Here the collective-spin variable

$$
\boldsymbol{\sigma}=\frac{1}{\sqrt{N}} \sum_{i=1}^{N} \mathbf{S}_{i}
$$

represents the vector of instantaneous magnetization fluctuation. It is the $1 / \sqrt{N}$ terms in (11) which make that model nonintegrable for finite $N>2$ in the presence of anisotropy. In order to arrive at a closed set of equations for the collective spin variables $\sigma_{\alpha}$, we must sum Eqs. (11) over all sites, divide by $\sqrt{N}$ and take the limit $N \rightarrow \infty$ :

$$
\dot{\sigma}_{\alpha}=\left(J_{\gamma}-J_{\beta}\right) \sigma_{\gamma} \sigma_{\beta}, \quad \alpha \beta \gamma=\text { c.p. }(x y z) .
$$

In spite of its complete integrability, the determination of dynamic correlation functions for the two-sublattice $X Y Z$ model can be quite involved. Here we concentrate on the simplest case, the fully isotropic $X X X$ model $\left(J_{x}=J_{y}=J_{z} \equiv J\right)$. We determine the $T=\infty$ autocorrelation functions for the sublattice spins $\sigma_{A}, \sigma_{B}$ from explicit solutions of the equations of motion (6). The same method was previously used in our study of the equivalent-neighbor $X X Z$ model. ${ }^{1,7}$ In terms of the new variables

$$
\sigma_{\alpha} \equiv \sigma_{A}^{\alpha}+\sigma_{B}^{\alpha}, \quad \tau_{\alpha} \equiv \sigma_{A}^{\alpha}-\sigma_{B}^{\alpha},
$$

Eqs. (6) turn (for this case) into two decoupled vector equations:

$$
\dot{\boldsymbol{\sigma}}=0, \quad \dot{\boldsymbol{\tau}}=J \boldsymbol{\tau} \times \boldsymbol{\sigma} .
$$

Both vectors have fixed length: $\tau^{2}=\tau_{x}^{2}+\tau_{y}^{2}+\tau_{z}^{2}=$ const and $\sigma^{2}=\sigma_{x}^{2}+\sigma_{y}^{2}+\sigma_{z}^{2}=$ const. The vector $\tau$ rotates uniformly about the stationary vector $\sigma$ with frequency $J \sigma$. We can then evaluate the collective-spin autocorrelation function $\left\langle\tau_{\alpha}(t) \tau_{\alpha}\right\rangle$ by using the solution of (15) and performing the ensemble average in two steps. In the expression

$$
\left\langle\tau_{\alpha}(t) \tau_{\alpha}\right\rangle=\frac{1}{3}\left\langle\left(\tau^{2}-\tau_{\alpha}^{2}\right) \cos (J \sigma t)\right\rangle+\frac{1}{3}\left\langle\tau_{\alpha}^{2}\right\rangle
$$

we have already carried out the time average over one period of the dynamical variable. The ensemble average is completed by averaging over the invariants $\sigma, \tau$, and $\tau_{\alpha}$. For that we need to know the joint probability distribution $P\left(\sigma, \tau, \tau_{\alpha}\right)$. In order to determine this distribution we use the statistical independence of the variables $\sigma_{A}^{\alpha}, \sigma_{B}^{\beta}: P\left(\sigma_{A}^{\alpha}, \sigma_{B}^{\beta}\right)=P\left(\sigma_{A}^{\alpha}\right) P\left(\sigma_{B}^{\beta}\right)$. Moreover, in the limit $N_{A}, N_{B} \rightarrow \infty$, we have

$$
P\left(\sigma_{A}^{\alpha}\right)=\sqrt{3 / 2 \pi} \exp \left[-\frac{3}{2}\left(\sigma_{A}^{\alpha}\right)^{2}\right] \text { and } A \rightarrow B,
$$

a result dictated by the central limit theorem. It follows that for $N_{A}, N_{B} \rightarrow \infty$ the variables $\sigma_{\alpha}, \tau_{\alpha}$ are also statistically independent and characterized by Gaussian distributions:

$$
\widehat{P}\left(\sigma_{\alpha}\right)=\sqrt{3 / 4 \pi} \exp \left(-\frac{3}{4} \sigma_{\alpha}^{2}\right) \text { and } \sigma_{\alpha} \rightarrow \tau_{\alpha} .
$$

Hence the distribution $P\left(\sigma, \tau, \tau_{\alpha}\right)$ factorizes into the distribution $\bar{P}(\sigma)$ of the length of vector $\sigma$ and the joint probability distribution $\bar{P}\left(\tau, \tau_{\alpha}\right)$. The former is evidently a Maxwellian:

$$
\bar{P}(\sigma)=4 \pi(3 / 4 \pi)^{3 / 2} \sigma^{2} \exp \left(-\frac{3}{4} \sigma^{2}\right)
$$

and the latter is given by the expression ${ }^{1,7}$

$\bar{P}\left(\tau, \tau_{\alpha}\right)=2 \pi(3 / 4 \pi)^{3 / 2} \tau \exp \left(-\frac{3}{4} \tau^{2}\right) \Theta\left(\tau-\left|\tau_{\alpha}\right|\right)$.

The evaluation of expression (16) with these distribution functions yields the following result for the collective-spin autocorrelation function:

$$
\left\langle\tau_{\alpha}(t) \tau_{\alpha}\right\rangle=\frac{2}{9}+\frac{4}{9}\left(1-\frac{2}{3} J^{2} t^{2}\right) \exp \left(-J^{2} t^{2} / 3\right) .
$$

The autocorrelation function for the sublattice spins $\sigma_{A}$, $\sigma_{B}$ inferred from (21) and the trivial result $\left\langle\sigma_{\alpha}(t) \sigma_{\alpha}\right\rangle=\frac{2}{3}$, reads

$$
\begin{aligned}
\left\langle\sigma_{A}^{\alpha}(t) \sigma_{A}^{\alpha}\right\rangle & =\left\langle\sigma_{B}^{\alpha}(t) \sigma_{B}^{\alpha}\right\rangle \\
& =\frac{2}{9}+\frac{1}{9}\left(1-\frac{2}{3} J^{2} t^{2}\right) \exp \left(-J^{2} t^{2} / 3\right) .
\end{aligned}
$$

The associated spectral density

$$
\Phi_{A}^{\alpha \alpha}(\omega)_{\sigma} \equiv \int_{-\infty}^{+\infty} d t e^{i \omega t} \frac{\left\langle\sigma_{A}^{\alpha}(t) \sigma_{A}^{\alpha}\right\rangle}{\left\langle\sigma_{A}^{\alpha} \sigma_{A}^{\alpha}\right\rangle},
$$

is then the sum of a $\delta$ function at $\omega=0$ and a Maxwellian spectral-weight distribution:

$$
\begin{aligned}
\Phi_{A}^{\alpha \alpha}(\omega)_{\sigma}= & (4 \pi / 3) \delta(\omega) \\
& +\frac{\pi}{2} \sqrt{4 \pi / 3}\left(\omega^{2} / J^{3}\right) \exp \left(-3 \omega^{2} / 4 J^{2}\right) .
\end{aligned}
$$

According to our classification of dynamical behavior, the spectral density (24) of the two-sublattice $X X X$ model 
belongs to the universality class $\lambda=1$ (Gaussian decay). The spectral densities of the equivalent-neighbor $X X X$ model belong to the same universality class. But the two models (2) and (3) part company when we reduce the rotational symmetry by introducing a uniaxial anisotropy $\left(J_{x}=J_{y} \neq J_{z}\right)$. For the spectral densities of the equivalent-neighbor $X X Z$ model, the universality class stays the same $(\lambda=1$, Gaussian decay), whereas it changes to $\lambda=3$ (stretched exponential decay) for those of the two-sublattice $X X Z$ model. When we further reduce the symmetry by introducing a biaxial anisotropy (e.g., $0<J_{x}<J_{y}<J_{z}$ ), we find that both models belong to the same universality class again $(\lambda=3)$. The remaining two universality classes mentioned at the beginning of this paper, $\lambda=0$ (compact support) and $\lambda=2$ (exponential decay), are realized in either model for finite $N$.

Our evidence for these realizations of universality classes of dynamical behavior is fairly complete in the case of the equivalent-neighbor $X Y Z$ model. It is based in part on an exact analysis and in part on results from applications of the recursion method. ${ }^{1}$ For the twosublattice $X Y Z$ model, the evidence is still fragmentary, based on an exact analysis that is at present incomplete for all but the simplest case.

The point we wish to emphasize in conclusion is the following. In both models (2) and (3), the reason for any change in universality class of dynamical behavior is associated with a switch between finite $N$ and infinite $N$ or a switch between linear dynamics and nonlinear dynamics. This mechanism allows for four different decay laws (1) of spectral densities, characterized by four different integervalued exponents $\lambda$.

The work reported here was supported by the U.S. National Science Foundation Grant No. DMR-90-07540.
${ }^{1}$ J.-M. Liu and G. Müller, Phys. Rev. A 42, 5854 (1990).

${ }^{2}$ C. Kittel and H. Shore, Phys. Rev. 138, A1165 (1965).

${ }^{3}$ R. Dekeyser and M. H. Lee, Phys. Rev. B 19, 265 (1979); M. H. Lee, I. M. Kim, and R. Dekeyser, Phys. Rev. Lett. 52, 1579 (1984).

${ }^{4}$ R. Dekeyser and M. H. Lee, Phys. Rev. B 43, 8123 (1991); 43,
8131 (1991).

${ }^{5}$ E. Magyari, H. Thomas, R. Weber, C. Kaufman, and G. Müller, Z. Phys. B 65, 363 (1987).

${ }^{6}$ N. Srivastava, C. Kaufman, G. Müller, R. Weber, and H. Thomas, Z. Phys. B 70, 251 (1988).

${ }^{7}$ J.-M. Liu and G. Müller, J. App1. Phys. 67, 5489 (1990). 